Retraction

\title{
Retracted: The Studies of Chlorogenic Acid Antitumor Mechanism by Gene Chip Detection: The Immune Pathway Gene Expression
}

\author{
Journal of Analytical Methods in Chemistry \\ Received 10 November 2020; Accepted 10 November 2020; Published 21 December 2020 \\ Copyright (c) 2020 Journal of Analytical Methods in Chemistry. This is an open access article distributed under the Creative \\ Commons Attribution License, which permits unrestricted use, distribution, and reproduction in any medium, provided the \\ original work is properly cited.
}

Journal of Analytical Methods in Chemistry has retracted the article titled "The Studies of Chlorogenic Acid Antitumor Mechanism by Gene Chip Detection: The Immune Pathway Gene Exdmpression" [1]. Confidential information from Sichuan Jiuzhang Biological Science \& Technology Co., Ltd. (previously known as Sichuan Jiuzhang Biochemical Engineering Science and Technology Development Co., Ltd.) was published without permission, as confirmed by a court judgment. A previous corrigendum that acknowledged the involvement of the company was not sufficient [2].

\section{References}

[1] T. Y. Kang, H. R. Yang, J. Zhang et al., "The Studies of Chlorogenic Acid Antitumor Mechanism by Gene Chip Detection: The Immune Pathway Gene Expression," Journal of Analytical Methods in Chemistry, vol. 2013, Article ID 617243, 7 pages, 2013.

[2] T. Y. Kang, H. R. Yang, J. Zhang et al., "Corrigendum to "The Studies of Chlorogenic Acid Antitumor Mechanism by Gene Chip Detection: The Immune Pathway Gene Expression"," Journal of Analytical Methods in Chemistry, vol. 2015, Article ID 538539, 1 page, 2015. 


\title{
The Studies of Chlorogenic Acid Antitumor Mechanism by Gene Chip Detection: The Immune Pathway Gene Expression
}

\author{
Tian Yi Kang, ${ }^{1}$ Hua Rong Yang, ${ }^{2}$ Jie Zhang, ${ }^{2}$ Dan Li, ${ }^{1}$ Jie Lin, ${ }^{1}$ Li Wang, ${ }^{1}$ and XiaoPing Xu${ }^{1}$ \\ ${ }^{1}$ West China School of Pharmacy, Sichuan University, Chengdu 610041, Sichuan Province, China \\ ${ }^{2}$ Jiuzhang Biochemical Engineering Science and Technology Development Co., Ltd, Chengdu 610041, Sichuan Province, China
}

Correspondence should be addressed to XiaoPing Xu; xu106@sina.com

Received 31 January 2013; Accepted 4 April 2013

Academic Editor: Yu-Ming Fan

Copyright (C 2013 Tian Yi Kang et al. This is an open access article distributed under the Creative Commons Attribution License, which permits unrestricted use, distribution, and reproduction in any medium, provided the original work is properly cited.

Persistently increasing incident of cancer in human beings has served to emphasize the importance of studies on mechanism of antitumor substances. Chlorogenic acid (CA), extracted from folium cortex eucommiae, has been confirmed to have lots of biological activities encompassing inhibition of tumor. However, the anticancer mechanism of CA remains unclear. Here, we have utilized a whole mouse genome oligo microarray $(4 * 44 \mathrm{~K})$ to analyze gene expression level of female BALB/c mice (implanted with EMT-6 sarcoma cells) after treatment with low, medium, and high-dose CA (5 mg/kg, $10 \mathrm{mg} / \mathrm{kg}$, and $20 \mathrm{mg} / \mathrm{kg}$ ), docetaxel, interferon, and normal saline separately at 6 time points (3rd, 6th, 9th, 12th, 15th, and 18th days after administration). Differentially expressed genes screened out by time-series analysis, GO analysis, and pathway analysis, and four immune-related genes were selected for further confirmation using RT-qPCR. The results demonstrated that CA is able to change gene expression and that the responsive genes (CaN, NFATC2, NFATC2ip, and NFATC3) involved in immune pathways had been significantly upregulated by CA. Expression of immune factors such as IL-2R and IFN- $\gamma$ can be improved by CA to promote activation and proliferation of $\mathrm{T}$ cells, macrophages, and NK cells, thus enhancing their surveillance and killing abilities, further suppressing the growth rate of tumor cells.

\section{Introduction}

Cancer dormant cell theory takes the view that activation of resting cells is the key factor causing cancer metastasis. Recent studies indicate that resting cells can be activated through escaping immune surveillance when immune function is weakened and that energy used for cell revivals can be provided by the newborn vessels [1-3]. Therefore, the study on improving body immune surveillance and restraining the energy of tumor growth will be an emergent mission as well as a breakthrough in the field of clinical treatment of cancer.

Chlorogenic acid (CA), extracted from folium cortex eucommiae and the flower bud of Lonicera confusa, is a kind of depside formed by caffeic acid and quinic acid. A large number of studies on CA have demonstrated that CA has a wide range of biological activities including inhibition of tumor cells [4]. According to preliminary studies, we recognize that $\mathrm{CA}$ is able to inhibit tumor in mouse except for these with T-cells defect, which suggests that immune system can be one of the targets of tumor suppression. Meanwhile, in vivo studies of our group indicate that $\mathrm{CA}$ also changes the advantage state of Th2 drift of BALB/c EMT- 6 mice. It significantly enhance the activities of BALB/c EMT- 6 mice cytotoxic T lymphocyte and natural killer cells as well as strengthening macrophage phagocytosis activity and lymphocyte transcription activity, thus boosting specific and nonspecific cellular immune function to tumor cells. Recent studies show that antitumor property of CA may have a connection with its ability of enhancing the activities of aryl hydrocarbon hydroxylase, suppressing formation of 8$\mathrm{OH}-\mathrm{dG}$, carcinogen-DNA adduct, and oxygen radical $[5,6]$. Meanwhile, CA can guard against gastric cancer and colon cancer and even suppress related carcinogenic factors $[7,8]$. In vitro studies revealed that CA can enhance T-cell proliferation caused by influenza virus antigen and can induce the generation of IFN- $\gamma$ and IFN- $\alpha$ by human lymphocytes and peripheral blood leukocytes $[9,10]$. Additionally, we find that $\mathrm{CA}$ can also activate neurocalcin to strengthen the activity 
of macrophagocyte [11]. Although much evidence has proved the anticancer property of CA, little is known about its exact targets on molecular level.

Base on the principle of complementary base pairing, microarray technology can distinguish particular genes from the mixture of genes by taking advantage of gene probes. Different from normal PCR, RT-qPCR can take quantitative analysis of unknown system. Meanwhile, its sensitivity, accuracy, and specificity are better than those of normal PCR. To explore the expression level of genes in tumor cells, we utilized a microarray technique to detect BALB/c EMT-6 mice after treatment with CA, docetaxel, interferon, normal saline separately, and the differences in expression level were confirmed by RT-qPCR. Time-series analysis, GO analysis, and pathway analysis were used to screen out common genes and analyze the relationship between putative genes and anticancer process of CA. Our data has suggested that CA is able to inhibit the growth of tumor through regulating immune system.

\section{Materials and Methods}

2.1. Animal Model Construction and CA Treatment. Female SPF mice (BALB/c) used in this experiment weigh 17-18 $\mathrm{g}$ on average, provided by the animal center of Sichuan University. EMT-6 sarcoma cells were provided by West China Hospital of Sichuan University Department of Health Engineering Key Laboratory of Transplantation and Transplantation Immunity.

We took EMT- 6 cell line out of the $-152^{\circ} \mathrm{C}$ ultra low temperature refrigerator. After thawing, centrifuging, and primary culturing, we used $0.25 \%$ trypsin for digestion twice and then subcultured it to a required number. All the collected cells were diluted with phosphate-buffered saline (PBS) in the end. Each BALB/c mouse was injected with $0.2 \mathrm{~mL}$ cell solution. The tumor would not transfer until it grew to a certain size. We homogenized the tumor taken from the body of BALB/c tumor-bearing mice to cell suspension and then inoculated the cell suspension to other BALB/c mice. Mice used for microarray analysis were injected with highdose CA (experimental group), docetaxel (control group), and normal saline (negative control group). Gene expression of experimental group was analyzed at six time points (3rd, 6 th, 9 th, 12th, 15th, and 18th days, resp., after administration), while the control group and negative control group were both analyzed at the 12th days after administration. RT-qPCR took mice injected with low, medium, and high-dose CA $(5 \mathrm{mg} / \mathrm{kg}$, $10 \mathrm{mg} / \mathrm{kg}, 20 \mathrm{mg} / \mathrm{kg}$ ) as experimental group, docetaxel and interferon as control group, and normal saline as negative control group. Each of these groups was tested 12 days after administration.

2.2. RNA Extraction and Labeling. Total RNA was extracted using mirVana RNA Isolation Kit (Applied Biosystem p/n AM1556) in 15 min after tissue collection. Quality and concentration of RNA were checked by spectrophotometer analysis and gel electrophoresis. All extracted samples had an A260/280 ratio of between 2.0 and 2.1 and a $28 \mathrm{~S} / 18 \mathrm{~S}$ ratio of 2 [12]. Agilent 2100 Bioanalyzer was used for further verification and qualified RNA had an RIN greater than 7.0. Total RNA was transcribed to double-stranded cDNA using RevertAid First Strand cDNA Synthesis Kit (Fermentas), with T7 Promoter Primer. The aaUTP-labeled cRNA was produced from cDNA by in vitro transcription. cRNA was dyed with Cy3 and purified using RNeasy Mini Kit (Qiagen, Valencia, CA, USA) according to the protocol of the RNeasy Plus Mini Kit.

2.3. Hybridization and Scanning. Cy3 cRNA must be fragmented in a buffer of $11 \mu \mathrm{L} 10 \times$ blocking agent, $2.2 \mu \mathrm{L} 25 \times$ fragmentation buffer and a certain amount of nuclease-free water at $60^{\circ} \mathrm{C}$ for half an hour before hybridization. We added $55 \mu \mathrm{L} 2 \times$ GEx hybridization buffer to the denatured and fragmented cRNA and then transfered them to microarrays at $65^{\circ} \mathrm{C}$ for $17 \mathrm{~h}$ with constant rotation. After hybridization, the microarrays were washed twice with buffer 1 for 1 minute followed by buffer 2 at $37^{\circ} \mathrm{C}$ for 1 minute too. In the end, the microarrays were autoscanned twice in $100 \%$ and $10 \%$ PMT, respectively, at $5 \mu \mathrm{m}$ resolution. The data was analyzed by Agilent Feature Extraction software and the quantile normalization was finished by GeneSpring 10.0.

2.4. Microarray Data Preprocessing. After acquisition of raw data, Agilent Feature Extraction software kit was used to eliminate the effect of background signals through automatic gridding. We took the $\log 2$ of the normalized background adjusted values to narrow the threshold of fluorescence signal strength before quantile normalization by GeneSpring 10.0 and then used a linear model to estimate expression values on the log scale. Both programs were available in the limma package. We chose a standard among the microarray samples thus the whole microarray data varied on it to obtain the same baseline of average density. Unqualified probes should be filtered out. Differentially expressed genes are submitted to Student's $t$-test and $P \leq 0.05$.

2.5. Interaction Network Analysis. We inputted the data of probes used for gene expressing analysis into SBC Analysis System (V2010.05).

2.6. RT-qPCR. RNA extracted from experimental group of $\mathrm{CA}$, positive control group of docetaxel, and interferon, negative control group were used to measure expression of selected genes of interest by RT-qPCR. Genes CaN, Nfatc2, Nfatc3, and Nfatc2ip showed a different expression level following treatment with low, medium, and high-dose CA, respectively. Primers used for RT-qPCR validation were designed according to each gene sequence and finished by SBC. Primer names, accession numbers, and sequences are listed in Table 1.

Total RNA was isolated using TRIzol reagents. The RNA integrity was verified with RNA formaldehyde electrophoresis and the quality was detected by spectrophotometry. The RNA samples had an A260/280 ratio of greater than 2 and a 20sRNA/18sRNA ratio of greater than 1.1. Single strand cDNA was synthesized using RevertAid First strand cDNA Synthesis 
TABle 1: Primers used for RT-qPCR validation and additional expression profiling.

\begin{tabular}{lccc}
\hline Gene & GenBank accession & Forward & Reverse \\
\hline Nfatc3 & NM_010901 & CTCCCTATCATAACCCA & CTgAAggCAAATCTgTG \\
Nfatc2ip & NM_010900 & AAAAgCAgAAAgCATACg & gACAggCCCTCCACTA \\
Nfatc2 & NM_010899 & TgAgAAgATCgTAggCAAC & gCTCgATgTCAgCgTTT \\
CaN & NM_001004025 & TgggAAATgAggCgATT & CCACgCTCAAAgAACCAg \\
\hline
\end{tabular}

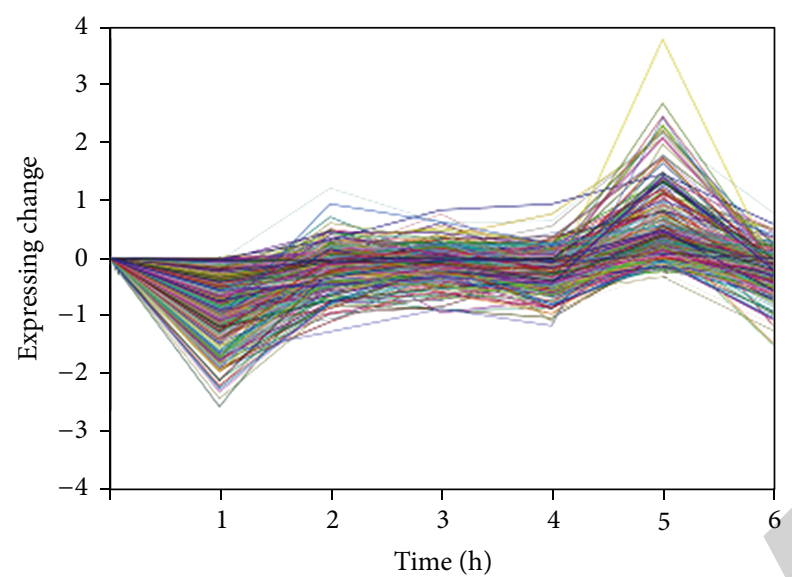

FIGURE 1: Expressing change of genes covered in NO.21 at 6 time points.

Kit. Reverse transcription reactions were conducted at $42^{\circ} \mathrm{C}$ for $30 \mathrm{~min}$ and at $85^{\circ} \mathrm{C}$ for $5 \mathrm{~min}$ at a $20 \mu \mathrm{L}$ total reaction volume containing the following reagents: $2 \mu \mathrm{L}$ RNA, $1 \mu \mathrm{L}$ oligo, $2 \mu \mathrm{L}(10 \mathrm{mM}) \mathrm{dNTP}$ mixture, $1 \mu \mathrm{L}$ RiboLock RNase Inhibitor, $4 \mu \mathrm{L} 5 \times$ reaction buffer, $9 \mu \mathrm{L}$ water, and $1 \mu \mathrm{L}$ RevertAid M-MuLV. PCR was performed using a Sino Bio Taq $2 \times$ Master Mix. The RT-qPCR products were analyzed by $1.0 \%$ agarose gel electrophoresis.

PCR reaction should be taken at a temperature gradient in the range of annealing temperature of $\mathrm{CaN}, \mathrm{Nfatc2}$, Nfatc3, and Nfatc2ip. RT-qPCR analysis was conducted to obtain crossing point $(\mathrm{Ct})$ values of each gene and standard curve was established through the linearity between $\mathrm{Ct}$ value and $\log 2$ of expression values. Before carrying out RT-qPCR analysis, cDNA and primers should be diluted in $20 \mu \mathrm{L}$ reagents containing the following: $9 \mu \mathrm{L}$ MIX, $8 \mu \mathrm{L} \mathrm{H} \mathrm{H}_{2} \mathrm{O}, 1 \mu \mathrm{L}$ sense primer (F), $1 \mu \mathrm{L}$ antisense primer $(\mathrm{R})$, and $1 \mu \mathrm{L}$ cDNA. The same cycling parameters were repeated for 40 times. Relative content of mRNA extracted from experimental group of CA, positive control group of docetaxel, and interferon negative control group was calculated by RT-qPCR detection system BIORAD IQ5.

\section{Results and Discussion}

3.1. Gene Expression Profiles of Tumor Cell after Being Treated by CA. 34275 probes without repeat were retained after combination and filtration. We carried out a Student's $t$-test of these probes to select differentially expressed genes $(P \leq$ $0.05)$ and then drew curves reflecting different expression
TABLE 2: Pathways of upregulated genes.

\begin{tabular}{lccc}
\hline Pathway name & Hits & Total & Percent (\%) \\
\hline $\begin{array}{l}\text { Natural killer cell mediated } \\
\text { cytotoxicity-Mus musculus (mouse) }\end{array}$ & 4 & 161 & 2.48 \\
$\begin{array}{l}\text { B-cell receptor signaling } \\
\text { pathway-Mus musculus (mouse) }\end{array}$ & 5 & 85 & 5.88 \\
$\begin{array}{l}\text { T-cell receptor signaling } \\
\text { pathway-Mus musculus (mouse) }\end{array}$ & 6 & 132 & 4.55 \\
\hline
\end{tabular}

TABLE 3: Upregulated genes in T-cell receptor signaling pathway, B-cell receptor signaling pathway, and natural killer cell mediated cytotoxicity.

\begin{tabular}{lccc}
\hline Pathway name & GeneID & ProbeID & Symbol \\
\hline Natural killer cell mediated & 18019 & Nfatc2 & NFATC2 \\
cytotoxicity_Mus musculus & 18021 & Nfatc3 & NFATC3 \\
(mouse) & 19059 & CaN & CAN \\
& 22325 & Vav2 & VAV2 \\
\hline & 17973 & Nck1 & NCK1 \\
& 56637 & Gsk3b & GSK3B \\
T-cell receptor signaling & 18019 & Nfatc2 & NFATC2 \\
pathway-Mus musculus (mouse) & 18021 & Nfatc3 & NFATC3 \\
& 19059 & CaN & CAN \\
& 22325 & Vav2 & VAV2 \\
\hline & 56637 & Gsk3b & GSK3B \\
& 18019 & Nfatc2 & NFATC2 \\
B-cell receptor signaling & 18021 & Nfatc3 & NFATC3 \\
pathway-Mus musculus (mouse) & 19059 & CaN & CAN \\
& 22325 & Vav2 & VAV2 \\
\hline
\end{tabular}

tendency of them at 6 time points. Eighty different curves were obtained after curve fitting. The smaller the $P$ value is, the more similar each gene trend fits the curve. NO.21 fitting curve had a $P$ value close to 0 and the expression profiles of genes covered in this fitting curve were analyzed systematically here. As shown in Figure 1, each inflection point corresponds to gene expression at different time points after administration. Genes covered in this curve were upregulated as seen from the whole time cycle; in other word, CA is capable of upregulating certain genes of tumor-bearing mice.

Data of genes covered in NO.21 was put into SBC Analysis System (V2010.05) and compared with KEGG (Kyoto Encyclopedia of Genes and Genomes) database. As shown in Table 2, 96 pathways are found to be related to upregulated genes and 29 of them are found to be statistically 
TABLE 4: GO of statistical significance.

\begin{tabular}{lccc}
\hline GO ID & Name & Hits & Total \\
\hline GO:0009055 & Electron carrier activity & 7 & 107 \\
GO:0003682 & Chromatin binding & 8 & 138 \\
GO:0046906 & Tetrapyrrole binding & 6 & 107 \\
GO:0030528 & Transcription regulator activity & 28 & 1053 \\
GO:0009058 & Biosynthetic process & 73 & 3388 \\
GO:0043167 & Ion binding & 78 & 3709 \\
\hline
\end{tabular}

significant including T-cell receptor signaling pathway, B-cell receptor signaling pathway, and natural killer cell mediated cytotoxicity. Immune-related genes expression is speculatedchanged after treatment with CA and probably have a trend of upregulation. Previous studies on CA immune function verified that CA could increase the carbon clearance index and the content of serum hemolysin and enhance the phagocytic function. Expression level of IFN- $\gamma$ and IL-2 increased whereas that for IL-4, IL-10 decreased. The release of tumor necrosis factor (TFN) was found reduced while the activation of cytotoxic T lymphocyte (CTL) which aimed at Lewis lung cancer was boosted. NK cells activation and lymphocyte transformation rate of Lewis lung cancer bearing mice were both significantly improved through the determination of immune function indexes. To sum up, CA has been proved capable of strengthening cellular immune functions to tumor. When 3 immune related pathways were found including upregulated genes, we inferred that the immunity of mouse had been improved after treatment of CA. GSK3B, NFATC2, NFATC3, CaN, VAV2, and NCK1 were found upregulated in the 3 immune related pathways as listed in Table 3. According to the functions, genes covered in NO.21 can be classified into 312 different GO (Gene Ontology) and 6 of them has a $P$ value less than 0.05 as listed in Table 4 . GO of statistical significance includes the following genes: Cyp2a4, Сyp2c37, Cyp2c38, Cyp27b1, Cyp51, E4f1, Fdps, Gata4, Eif2ak1, Myt1l, Rarg, Sod1, Sp1, Taf3, Nr2c2, Hnf4g, Zdhhc3, Phf6, Mynn, Zfp386, Zfp617, Zfp114, Zfp238, Zfp174, Zfp113, Zfp109, Zfp263, Pcdhb6, Pcdhb15, Pcdhb20, Pcdhb21, Cdyl, Nfatc3, Pbx2, Rest, Smarca5, and Nfatc2. After doing some queries on the function of genes covered in statistically significant pathways and GO, we found out immune related genes and common genes of pathway and GO. Nfatc2, Nfatc3, Nfatc2ip, and $\mathrm{CaN}$ were found through combing raw analysis data of gene expression profile, as shown in Table 5.

3.2. Validation of Differentially Expressed Genes by Fluorescence Quantitative PCR. Relative expression level of the target genes Nfatc2, Nfatc3, Nfatc2ip, and CaN on average is shown in Table 6. Relative expression level of Nfatc2, Nfatc3, Nfatc2ip, and CaN in high-dose CA group, Nfatc2ip and $\mathrm{CaN}$ in medium-dose $\mathrm{CA}$ group, and $\mathrm{CaN}$ in low-dose $\mathrm{CA}$ group significantly improved compared with negative control group. The exact expression quantity is shown in Figure 2.

As shown in Figure 2, relative expression level of Nfatc2ip, Nfatc3, Nfatc2, and CaN in high-dose CA group improved
TABLE 5: Immune related genes covered in GO and pathway.

\begin{tabular}{lcc}
\hline GenBank accession & GO ID & Gene symbol \\
\hline NM_010899 & GO:0001816 & Nfatc2 \\
NM_010900 & GO:0001816 & Nfatc2ip \\
NM_010901 & GO:0003677 & Nfatc3 \\
NM_001004025 & GO:0004723 & CaN \\
\hline & &
\end{tabular}

compared with negative control group, which corresponds to the result of microarray analysis.

3.3. Discussion of Genes Nfatc2ip, Nfatc3, Nfatc2, and CaN. Here, a whole mouse genome oligo microarray $(4 * 44 \mathrm{~K})$ was used to analyze gene expression level of female BALB/c mice and to compare the expression of corresponding target genes of each group on the time series. Systematic error or accidental error can affect the accuracy of microarray analysis to some extent and the errors may come from the processes of RNA extraction, RNA reverse transcription, and hybridization as well as the quality of gene chips and RNA. While the results of microarray analysis can reflect expressing pattern at body's internal gene level quickly, false positive results possibly caused by systematic error or accidental error must be taken into consideration. Verifying experiments like ELISA test, western blot test, and PCR test should be taken for further confirmation. Here, the results of PCR test help completing the study as well as making it more convincing. Through the analysis of genes included in NO.21, expression of critical genes in T-cell receptor signaling pathway, B-cell receptor signaling pathway, and natural killer cell mediated cytotoxicity had changed and immune related genes Nfatc2ip, Nfatc3, Nfatc2, and CaN were found.

NFAT (nuclear factor of activated T cells) which consists of 4 components NFATc1, NFATc2, NFATc3, and NFATc4 has been proved important in lymphocyte activation and development. Nfatc2 existing in cytoplasm translocates to the nucleus upon T-cell receptor stimulation and then becomes a member of the nuclear factor of activating T-cells transcription [13]. When the body lacks NFATc2, lymphocyte apoptosis will be significantly reduced which suggests lymphangiectasia and Th2-type response. Meanwhile, the Th1/Th2 balance will be destroyed which suggests a Th2 polarization. Immune regulatory function will disorder when Th1/Th2 balance is destroyed and tumor occurrence shows the preponderance state tendency of Th2. This state will weaken 


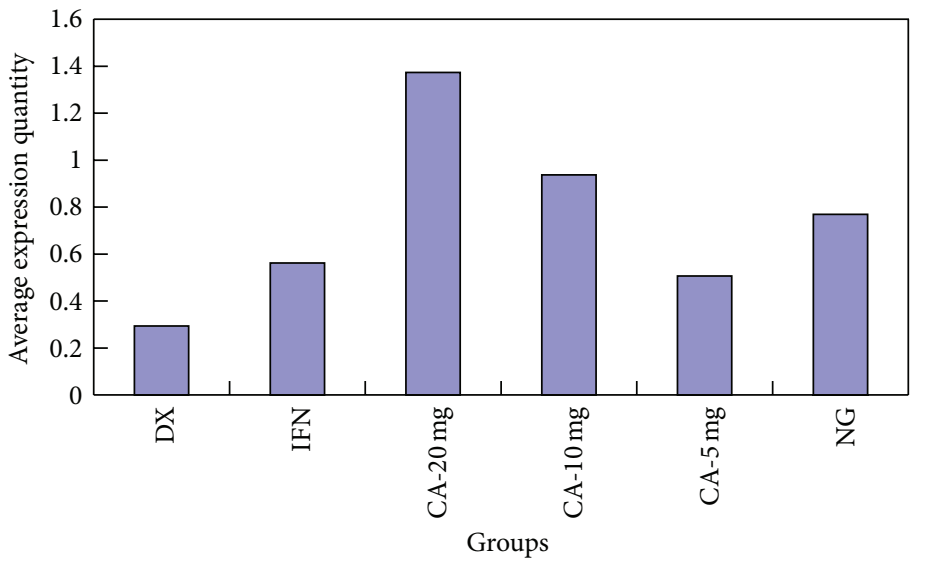

(a)

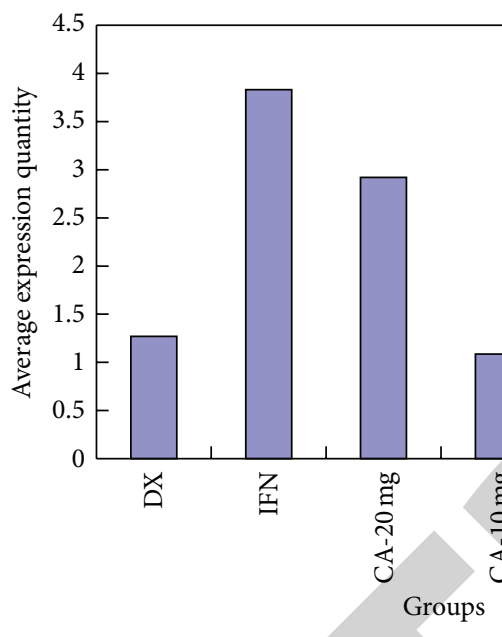

(c)

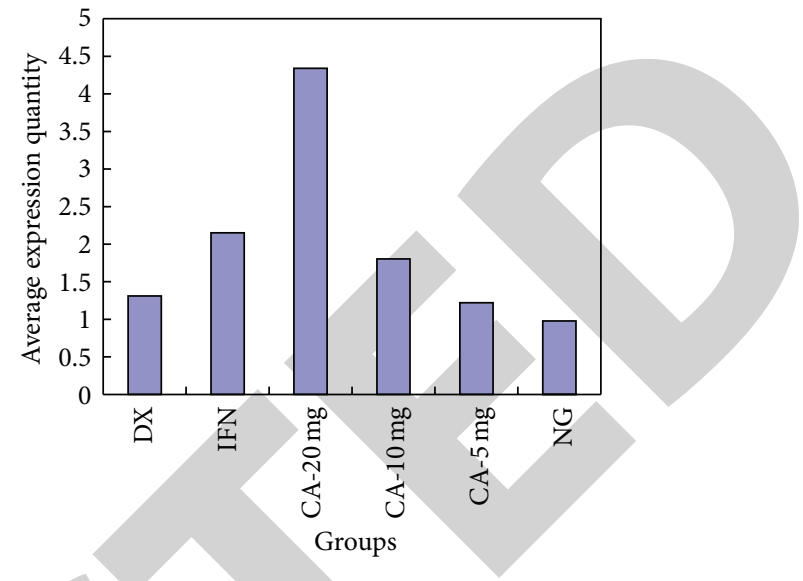

(b)

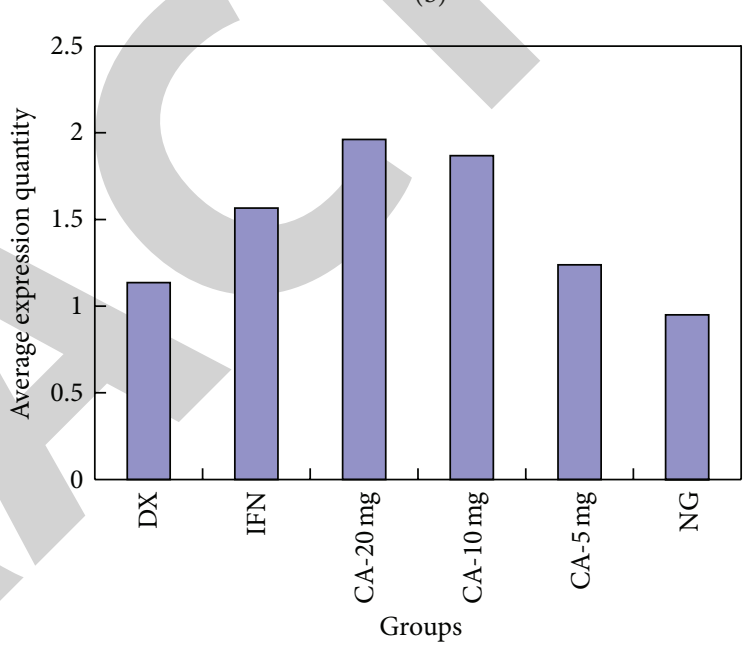

(d)

FIgURE 2: Relative expressing quantity of Nfatc2ip, Nfatc3, Nfatc2, and CaN in each groups. (a) Comparsion of Nfatc2ip expressing quantity among every group. (b) Comparsion of Nfatc3 expressing quantity among every group. (c) Comparsion of Nfatc 2 expressing quantity among every group. (d) Comparsion of CaN expressing quantity among every groups.

\begin{tabular}{|c|c|c|c|c|}
\hline \multirow{2}{*}{ Group } & \multicolumn{4}{|c|}{ Gene } \\
\hline & Nfatc2 & Nfatc3 & Nfatc2ip & $\mathrm{CaN}$ \\
\hline Docetaxel & $1.270 \pm 1.216$ & $1.311 \pm 0.573$ & $0.294 \pm 0.160$ & $1.136 \pm 0.544$ \\
\hline Interferon & $3.831 \pm 4.960$ & $2.152 \pm 1.089$ & $0.562 \pm 0.464$ & $1.566 \pm 0.914$ \\
\hline High-dose CA & $2.920 \pm 2.047$ & $4.341 \pm 3.961$ & $1.373 \pm 1.096$ & $1.961 \pm 0.927$ \\
\hline Mid-dose CA & $1.086 \pm 0.367$ & $1.803 \pm 1.255$ & $0.937 \pm 0.950$ & $1.868 \pm 1.160$ \\
\hline Low-dose CA & $1.185 \pm 1.156$ & $1.220 \pm 1.303$ & $0.507 \pm 0.168$ & $1.238 \pm 0.310$ \\
\hline Negative & $1.085 \pm 0.462$ & $0.979 \pm 0.839$ & $0.769 \pm 1.599$ & $0.950 \pm 0.418$ \\
\hline
\end{tabular}

antitumor immune function and will induce tumor cells free from immune surveillance and immune attack. This may be one of the immune mechanisms of tumor development and provides a new idea of tumor treating. The reverse of Th1/Th2 abnormal drift is in favor of recovering antitumor immunocompetence and reducing tumor recurrence and metastasis to improve long-term survival rate finally.
NFATc2 and NFATc3 have been proved to synergistically regulate the reaction of $\mathrm{T}$ cell receptor, cell division, and Th2 differentiation. Th2-type reaction which suggests the secretion of IL-4, IL-5, and IL- 6 is increased whereas the decrease IL-2, IFN- $\gamma$, TNF- $\alpha$, and IL-10 happens when the body lacks NFATc2 and NFATc3 [14-16]. When B cell and Tcell lack NFATc2 and NFATc3 simultaneously, the function 
of $\mathrm{T}$ cell will be weakened but the ability of $\mathrm{T}$ cell receptor mediating cell proliferation still exists, while $\mathrm{B}$ cells overactivate and show excessive differentiation [17]. The fact that expression of NFATc2 and NFATc3 is improved after treatment of CA indirectly indicates that CA could reverse Th1/Th2 drift. Meanwhile, secretion of IL-2, IFN- $\gamma$, TNF- $\alpha$, and IL-10 is connected to CA too. High expression of NFATc2 and NFATc3 is in favor of the secretion of these cytokines which have already been used as nonspecific immunity treatment to cancer. NFATc2ip can induce the expression of T-cell cytokines, especially enhancing IL production. Three splice variants existing in NFATc2ip are able to methylate NFATc2ip after its translation to produce NFATc2ip regulatory factor. Expression of Th1-type and Th2-type cell factors will be suppressed when the methylation process is inhibited. Therefore, methylation process of NFATc2ip is an important controlling point of manipulating expression of NFATdependent cell factors but it will not have any influence on general transcription factor of Thl and Th2 nor NFAT activation.

$\mathrm{CaN}$ (Calcineurin) is known as the only serine/threonine protein phosphatase regulated by $\mathrm{Ca}\left({ }^{2+}\right)$-calmodulin so far and mainly aims at catalyzing dephosphorylation of phosphatidylserine and phosphatidyl threonine. It is a multifunctional signaling enzyme involved in function regulation of many cells and distributes in a wide range of tissues especially nerve tissue, T lymphocytes, heart, and skeletal muscle [18, 19]. NFAT family is the main substrate of CaN. NFAT regulates many genes expression as well as influencing many cells differentiation through CaN/NFAT signaling pathway. $\mathrm{CaN}$ is able to make NFAT existing in cytoplasm move into cell nucleus after dephosphorylation to finish following transcription and translation, and then Nfatc can combine with AP-1 family members alone or in groups to stimulate secretion of cell factors in certain areas such as IL-2. Transcription induction of IL-2 is a sign of T-cells activation. As mentioned above, NFAT is very important in regulating immunoreaction; meanwhile, NFATc3 and NFATc2 play a particularly key role in correcting body's immune function. Thus, high expression of $\mathrm{CaN}$ is a benefit to dephosphorylation of NFAT and indirectly has an effect upon immune system.

\section{Conclusion}

The result of microarray analysis of differentially expressed genes of female BALB/c EMT- 6 mice has indicated that the antitumor mechanism of CA is closely related to body's immune system. Through upregulating, the expression of CaN, NFATC2, NFATC2ip, and NFATC3, CA is able to improve the transcription of immune factors like IL-2R and IFN- $\gamma$, stimulate proliferation and activation of T cells, NK cells, and macrophage, strengthen monitoring and killing abilities of cancer cells, and inhibit growth of tumor finally. The understanding of the anticancer mechanism of CA has provided a reliable evidence of taking advantage of CA to fight against cancer.

\section{Disclosure}

Hua Rong Yang and Jie Zhang, employed by Jiuzhang Biochemical Engineering Science and Technology Development Co., Ltd, are cooperators of this project. All of the other authors are researchers of West China School of Pharmacy, Sichuan University, and have no conflict of interests. Reagents were supported by the fund (The Sichuan Province Science and Technology Support Project Fund 2011SZ0131) of this project. Some parts of the experiment were entrusted to the third party but not sponsored by it. This statement is made in the interest of full disclosure and not because the authors consider this to be a conflict of interests.

\section{Acknowledgments}

The Sichuan Province Science and Technology Support Project Fund 2011SZ0131 supported this work. The authors would like to thank Hongwei Liu (Shanghai Biochip Co., Ltd.) for his great help in microarray analysis.

\section{References}

[1] R. McKallip, R. Li, and S. Ladisch, "Tumor gangliosides inhibit the tumor-specific immune response," Journal of Immunology, vol. 163, no. 7, pp. 3718-3726, 1999.

[2] S. A. Rosenberg, "Progress in human tumour immunology and immunotherapy," Nature, vol. 411, no. 6835, pp. 380-384, 2001.

[3] S. Ostrand-Rosenberg, "Animal models of tumor immunity immunotherapy and cancer vaccines," Current Opinion in Immunology, vol. 16, no. 2, pp. 1430-1450, 2004.

[4] A. L. Zhang, Q. Ma, and J. M. Gao, "Studies on bioactivities of chlorogenic acid and its analogues," Chinese Traditional and Herbal Drugs, vol. 32, no. 2, pp. 173-176, 2001.

[5] H. Kasai, S. Fukada, Z. Yamaizumi et al., "Action of chlorogenic acid in vegetables and fruits as an inhibitor of 8-hydroxy-deoxyguanseine formation in vitro and in a rat carcinogenesis model," Food and Chemical Toxicology, vol. 38, no. 5, pp. 467-471, 2000.

[6] E. Ignatowicz, B. Balana, S. V. Vulimiri et al., "The effect of plant Phenolics on the formaiton of 7, 12-dimethylbenz[a] anthracene-DNA adducts and TPA- stimulated polymorphonuclear neutrophifls chemiluminescence in vitro," Toxicology, vol. 189, no. 3, pp. 199-209, 2003.

[7] M. Shimizu, N. Yoshimi, Y. Yamach et al., "Suppressive effects of chlorogenic acid on N-methyl-N-nitrosourea-indueed glandular stomach carcinogenesis in male F344 rats," Toxicological Sciences, vol. 24, no. 5, pp. 433-439, 1999.

[8] K. Matsunaga, M. Katayama, K. Sakata et al., "Inhibitory effects of chlorogenic acid on azoxymethane-induced colon carcinogenesis in male F344 rats," Asian Pacific Organization for Cancer Prevention, vol. 3, no. 2, pp. 163-166, 2002.

[9] L. C. Chiang, L. T. Ng, W. Chiang et al., "Immunomodulatory activities of flavonoids, monoterponoids, triterpenoids, iridoid glycosides and phenolic compounds of Plantago species," Planta Medica, vol. 69, no. 7, pp. 600-604, 2003.

[10] K. J. Hu, F. J. Qu, Z. L. Wang et al., "Experimental study on the induction of a-tinterferon of human leucocyte in vitro by chlorogenic acid," Journal of Harbin Medical University, vol. 38, pp. 120-122, 2004.

[11] H. Z. Wu, J. Luo, Y. X. Yin et al., "Effects of chlorogenic acid, an active compound activating calcineurin, purified from Flos 
Lonicerce on macrophage," Acta Pharmacologica Sinica, vol. 25, no. 12, pp. 1685-1689, 2004.

[12] P. D. Rogers and K. S. Barker, "Evaluation of differential gene expression in fluconazole-susceptible and -resistant isolates of Candida albicans by cDNA microarray analysis," Antimicrobial Agents and Chemotherapy, vol. 46, no. 11, pp. 3412-3417, 2002.

[13] S. Baksh, H. R. Widlund, A. A. Frazer-Abel et al., "NFATc2mediated repression of cyclin-dependent kinase 4 expression," Molecular Cell, vol. 10, no. 5, pp. 1071-1081, 2002.

[14] M. R. Hodge, A. M. Ranger, F. C. de la Brousse, T. Hoey, M. J. Grusby, and L. H. Glimcher, "Hyperproliferation and dysregulation of IL-4 expression in NF-ATp-deficient mice," Immunity, vol. 4, no. 4, pp. 397-405, 1996.

[15] A. Kiani, J. P. B. Viola, A. H. Lichtman, and A. Rao, "Downregulation of IL-4 gene transcription and control of Th2 cell differentiation by a mechanism involving NFAT1," Immunity, vol. 7, no. 6, pp. 849-860, 1997.

[16] A. M. Ranger, M. R. Hodge, E. M. Gravallese et al., "Delayed lymphoid repopulation with defects in IL-4-driven responses produced by inactivation of NF-ATc," Immunity, vol. 8, no. 1, pp. 125-134, 1998.

[17] K. Schuh B Kneitz J Heyer et al., "Retarded thymic involution and massive germinal center formation in NF-ATp-deficient mice," The European Journal of Immunology, vol. 28, no. 8, pp. 2456-2466, 1998.

[18] H. Mukai, C. D. Chang, H. Tanaka, A. Ito, T. Kuno, and C. Tanaka, "cDNA cloning of a novel testis-specific calcineurin Blike protein," Biochemical and Biophysical Research Communications, vol. 179, no. 3, pp. 1325-1330, 1991.

[19] K. L. Dodge and J. D. Scott, "Calcineurin anchoring and cell signaling," Biochemical and Biophysical Research Communications, vol. 311, no. 4, pp. 1111-1115, 2003. 